\title{
The Effects of Doses and Methods of Lime Placement to N, P, K, Ca, Mg Content into the Leaves and Sugarcane Growth in Ultisol Seputih Mataram Lampung Tengah
}

\author{
Heri Wahyudi*, Azwar Maas, Eko Hanudin, Sri Nuryani Hidayah Utami \\ Department of Soil, Faculty of Agriculture Universitas Gadjah Mada \\ Jln. Flora no. 1, Bulaksumur, Sleman, Yogyakarta 55281, Indonesia \\ *Corresponding email: heriwahyudi22@yahoo.co.id
}

\section{Received: 08 $8^{\text {th }}$ November 2017; Revised: $11^{\text {st }}$ December 2017; Accepted: $08^{\text {th }}$ February 2018}

\begin{abstract}
Land circumstances on Seputih Mataram, Lampung included in dried area consisted of ultisols soil. Ultisol has pH less than 5.5 caused the amount of soil nutrient absorbed to be not optimal yet. This research aimed to know the effect of adding dolomite to the soil chemical content ( $\mathrm{pH}$ of $\mathrm{H}_{2} \mathrm{O}, \mathrm{KCL}$, content of $\mathrm{N}, \mathrm{P}, \mathrm{K}, \mathrm{Ca}, \mathrm{Mg}, \mathrm{Organic}-\mathrm{C}, \mathrm{C} / \mathrm{N}$, Al-exc, H-exc, CEC), nutrient content on the leaves (N, P, K, Ca, Mg), and sugar cane physic. This research used Completely Randomized Design in 2 factors with 3 replication. The first factor was the dolomite dosses with 6 levels: without the dolomite (K0), $500 \mathrm{~kg} \mathrm{ha}^{-1}(\mathrm{~K} 1), 1,000 \mathrm{~kg} \mathrm{ha}^{-1}(\mathrm{~K} 2), 2,000 \mathrm{~kg} \mathrm{ha}^{-1}(\mathrm{~K} 3), 4,000 \mathrm{~kg} \mathrm{ha}^{-1}$ (K4), and 6,000 kg ha-1 (K5). The second factor (R) was the depth of dolomite application: (R1) dolomite with 0-20 cm deep, and (R2) dolomite with 20-40 cm deep. The observation was done to know the soil chemical content, the leaves nutrients, and the variables that could be used into the research. The result showed that the depth of dolomite did not have significant effect to the amount of Nitrogen (N), Phosphorus (P), Potassium (K), Calcium (Ca), and Magnesium (Mg) in leaves. Dolomite addition had significant effect for Calcium $(\mathrm{Ca})$ absorption in leaves, but it did not significantly affect the leaves absorption. Dolomite addition had significant effect in the leaves content and Magnesium (Mg) leaves absorption. $6,000 \mathrm{~kg} \mathrm{ha}^{-1}$ dolomite for physical soil characteristic ( $\mathrm{pH} \mathrm{H} \mathrm{H}_{2} \mathrm{O}, \mathrm{pH} \mathrm{KCL}, \mathrm{C} / \mathrm{N}$ ratio, $\mathrm{K}, \mathrm{P}, \mathrm{Ca}, \mathrm{Mg}$, Exch-H), leaves nutrient $(\mathrm{N}, \mathrm{K}, \mathrm{Ca}), \mathrm{Mg}$ and $\mathrm{Ca}$ absorption on leaves also sugar cane tonnage per hectare showed the maximum result.
\end{abstract}

Keywords: Dolomite, dry matter, liming, sugarcane, ultisol

\section{INTRODUCTION}

Sugar is one of basic needs of most people all over the world and the demand for sugar shows more increase annually. In addition, sugar is also important for industries. Sugar is currently becoming a problem due to the lack of domestic sugar production, while the demand continues to increase. To meet the domestic sugar demand and reduce sugar import, the production of sugar raw material which is sugarcane should be increased.

The Sugar Group Companies land condition belongs to a dry land (soil taxonomy) with Ultisol soil type. Ultisol is often associated with infertile soil, but in fact it is potential to be used for agricultural land in proper managements which pay attention to the existing problems (constraints). Ultisol can also be a potential land if the climate is supporting. Ultisol has $\mathrm{pH}$ of less than 5.5. The soil $\mathrm{pH}$ is determinant in controlling other chemical soil properties and characteristics for soil fertility.

One of efforts in improving sugarcane productivity is by intensification (increasing sugarcane production per unit of land area). The most common intensification methods are good land processing, balanced fertilizing, using superior varieties, selecting proper time for planting and harvesting, and proper weed and pest controlling.

Another problem of intensification is the need of manpower in agriculture which is more difficult to get from time to time, because manpower is more expensive and less available. This manpower is required for land management activities such as for applying fertilizer. New breakthroughs are required to solve 
the problems and these include mechanization and efficiency of liming.

Liming in PT. Gula Putih Mataram so far has been practiced manually by broadcasting on the soil surface and using fertilizer sowing. Applied dose uses common guideline for sugarcane fertilizing, so that it is possible to use slot application and the problem of mechanization is overcome. Moreover, considering that calcium is a soil macro nutrient which also affects $\mathrm{pH}$ neutralization that takes a role to improve other nutrient uptakes, the slot application into particular depths will stimulate sugarcane rooting. By better rooting, it is expected that soil nutrient uptakes will be more optimal to improve sugarcane productivity. Therefore, the objectives of the present study were (i) to determine optimal doses of dolomite to sugarcane growth and (ii) to determine the right dolomite placement for sugarcane plant growth.

\section{MATERIALS AND METHODS}

This research was conducted in sugarcane plantation area of Division IV, block TS 06/03, plot 024TS060 of PT. Gula Putih Mataram, located in Mataram Udik Village, Bandar Mataram Sub

District, Central Lampung Regency, Lampung Province. The experiment was conducted from May 2016 to January 2017. Materials and equipment used were sugarcane germ variety MLR-236, land processing equipment (tractor and its supporting equipment), lime immersion equipment, dolomite, stationaries, scales, roll meter, wooden pegs, ropes, and chemical materials for laboratory analysis.

Land preparation included land processing of stillage, brushing, plough, harrow I and II, track making, ripping, immersed dolomite application by using implementer, and then followed by furrowing when 1 MAA (with TSP $100 \mathrm{~kg} \mathrm{ha}^{-1}$ and ZA $100 \mathrm{~kg}$ $\mathrm{ha}^{-1}$ ) and irrigation (when there was no rain). After 3 MAA top dressing application used Urea $283 \mathrm{~kg} \mathrm{ha}^{-1}$. Sugarcane planting by immersing was conducted with double rows planting pattern (1 row contains of two lines). The plot measure for each treatment was 2 rows $\times 150 \mathrm{~m}$.

This research used completely randomized design with two factors and three blocks for repetitions. The first factor was the dolomite in six doses: without dolomite (K0), $500 \mathrm{~kg} \mathrm{ha}^{-1}(\mathrm{~K} 1), 1.000 \mathrm{~kg} \mathrm{ha}^{-1}(\mathrm{~K} 2)$, $2,000 \mathrm{~kg} \mathrm{ha}^{-1}(\mathrm{~K} 3), 4,000 \mathrm{~kg} \mathrm{ha}^{-1}(\mathrm{~K} 4)$, and $6,000 \mathrm{~kg}$ $\mathrm{ha}^{-1}(\mathrm{~K} 5)$. The second factor was the depth of placement of dolomite application: application in $0-20 \mathrm{~cm}$ of soil depth (R1) and dolomite application in 20-40 $\mathrm{cm}$ of soil depth (R2). There were total of

12 combination treatments $(6 \times 2)$. Each treatment was repeated three times so that there were 36 plots $(3 \times 12)$.

Initial data collections in this research were soil physical properties (soil texture), soil chemical properties, and dolomite analysis. I nitial soil sampling and 7 MAA soil sampling were conducted in soil layer of $0-20 \mathrm{~cm}$ and $20-40 \mathrm{~cm}$. The soil chemical properties analysis included soil $\mathrm{pH}\left(\mathrm{pH} \mathrm{H}_{2} \mathrm{O} ; \mathrm{pH}\right.$ $\mathrm{KCl}$ ), $\mathrm{N}, \mathrm{P}, \mathrm{K}, \mathrm{Ca}, \mathrm{Mg}$, organic- $\mathrm{C}$ contents, $\mathrm{C} / \mathrm{N}$ ratio, Al-exc, $\mathrm{H}$-exc and cation exchange capability (CEC). All analysis activities for soil and nutrient uptakes were conducted in the Agrolab laboratory of PT. Gula Putih Mataram. Leaf nutrient content analysis was conducted at $7 \mathrm{MAA}$ and it included $\mathrm{N}$, $\mathrm{P}, \mathrm{K}, \mathrm{Ca}$, and $\mathrm{Mg}$ content. Other observation parameters were effects on sugarcane performance involving sugarcane population and height from $2 \mathrm{MAA}$ to 7 MAA, wet and dry biomass at 7 MAA and sugarcane weight per hectare at 7 MAA.

These data were analyzed to find out the effects of the independent variables to dependent variable with analysis of variance by using F-test at $5 \%$ of trust content, and when there were significant differences between treatments, it was followed by DMRT (Duncan's Multiple Range Test) at 5\% of trust content (Gomez and Gomez, 1993). Data analysis was conducted with SAS 9.1.3 Portable software.

\section{RESULTS AND DISCUSSION}

The key for symbiotic between mycorrhiza and host plant was the infection capability of the mycorrhiza (Smith et al., 2011). Initial soil analysis is presented in soil had a very low organic matter content Table 1. This table shows that soil (organic-C $0.51 \%$ and $0.38 \%$ ) and texture in research location was together with low $\mathrm{C} / \mathrm{N}$ ratio it showed that dominated by sand fraction (sand $66-68 \%$, the soil organic matter was already at silt 5-6\% and clay 26-28\%) and this soil decomposed stage, suggesting that this soil texture was categorized as sandy requires organic material addition. Organic clayloam. According to Tambunan (2008) C as energy source in the soil was very sandy soil will have many macro pores. important for soil microorganism, so lack More porous soil will make roots easier to of this will decrease soil microbe population penetrate, and easier for water and air to and diversity which had an important role circulate, but water and air are also easier in transforming 
Table 1. Soil physical and chemical properties in the research location

\begin{tabular}{|c|c|c|c|c|}
\hline \multirow{3}{*}{ Soil Properties } & \multicolumn{4}{|c|}{ Soil depth } \\
\hline & \multicolumn{2}{|c|}{$0-20 \mathrm{~cm}$} & \multicolumn{2}{|c|}{$20-40 \mathrm{~cm}$} \\
\hline & $0-20 \mathrm{~cm}$ & Grade & $20-40 \mathrm{~cm}$ & Grade \\
\hline Texture & Value & Category* & Value & Category* \\
\hline Sand (\%) & 68 & & 66 & \\
\hline Silt (\%) & 5 & Clay with sand & 6 & Clay with sand \\
\hline Clay $(\%)$ & 26 & & 28 & \\
\hline \multicolumn{5}{|l|}{$\mathrm{pH}$} \\
\hline $\mathrm{H}_{2} \mathrm{O}$ & 4.67 & Acid & 4.52 & Acid \\
\hline $\mathrm{KCl}$ & 4.46 & Very acid & 4.28 & Very acid \\
\hline \multicolumn{5}{|l|}{ Organic materials } \\
\hline C-Organic (\%) & 0.51 & Very low & 0.38 & Very low \\
\hline Total N (\%) & 0.09 & Very low & 0.07 & Very low \\
\hline $\mathrm{C} / \mathrm{N}$ & 5.65 & Low & 5.46 & Low \\
\hline Available P (ppm) & 18.44 & Very high & 4.46 & Low \\
\hline Exch-K (me/100 g soil) & 0.03 & Very low & 0.04 & Very low \\
\hline Exch-Ca (me/100 g soil) & 1.01 & Very low & 1.07 & Very low \\
\hline Exch-Mg (me/100 g soil) & 0.22 & Very low & 0.25 & Very low \\
\hline Exch-Al (me/100g) & 0.49 & & 0.34 & \\
\hline Exch-H (me/100g) & 0.52 & & 0.51 & \\
\hline \multicolumn{5}{|l|}{ Cation Exchange } \\
\hline Capacity (me/100g) & 3.61 & Very low & 4.97 & Very low \\
\hline
\end{tabular}

Table 2. Physical and chemical properties of dolomite used in this research

\begin{tabular}{lcccc}
\hline Water content $(\%)$ & $\mathrm{CaO}(\%)$ & $\mathrm{MgO}$ & Mesh 25 & Mesh 80 \\
\hline 14.34 & 30.17 & 16.59 & 84 & 51 \\
\hline
\end{tabular}

varying soil nutrients to be depleted. This means that this soil (Santoso et al., 1999). Macro type has low capability in storing water. nutrient elements in the soil were very low Initial soil $\mathrm{pH}$ was in acid category with up to $40 \mathrm{~cm}$ of soil depth except for the $\mathrm{pH}$ of 4.67 and 4.54 , respectively for 0 - available $\mathrm{P}$ which was very high in $0-20 \mathrm{~cm}$ of soil depth. The $\mathrm{Ca}$ and $\mathrm{Mg}$ content were very low so that dolomite addition was required. The cation-exchange capacity very was low (3.61\% and $4.97 \%)$, so that the ion exchange was very low causing the soil assessment score to be low (Sutanto, 2005).

The water content of dolomite used in this research (Table 2) was higher than what was stipulated in SNI 02-2804-1992 causing the required dose to increase $1 \mathrm{me}^{100 \mathrm{~g}^{-1}}$ of soil. For the material dose, the dolomite used was middle category and this was related to $\mathrm{Ca}$ and $\mathrm{Mg}$ dissolution speed into the soil.

\section{Soil chemical properties at 7 MAA}

Table 3 showed that the dolomite doses affected significantly in increasing soil acidity $\mathrm{pH} \mathrm{H} 2 \mathrm{O}$ and $\mathrm{pH} \mathrm{KCl}, \mathrm{Mg}$, Al-exc and H-exc contents, but the soil application depths of dolomite by using lime immersing implementer did not significantly affect these parameters. Concerning organic-C content, total $\mathrm{N}, \mathrm{C} / \mathrm{N}$ ratio, available $\mathrm{P}$, available $\mathrm{K}, \mathrm{Ca}$, and $\mathrm{CEC}$, the dolomite doses did not have significant effect, but the dolomite immersion depths showed significant effect to those parameters. In all observed soil chemical properties, there were interactions between doses and dolomite immersion depths.

The lowest $\mathrm{pH} \mathrm{H} 2 \mathrm{O}$ score was in control (K0) with $20-40 \mathrm{~cm}$ of soil (R2) depth $\left(\mathrm{KOR}_{2}\right.$ score was 3.94) where this was in very acidic category and the highest score was obtained at $6,000 \mathrm{~kg} \mathrm{ha}^{-1}$ (K5) at $20-40 \mathrm{~cm}$ of soil depth $\left(\mathrm{K}_{5} \mathrm{R}_{2}\right.$ score was 5.65$)$ and this was moderate acidic category. $0-20 \mathrm{~cm}$ 
Table 3. Soil physical and chemical properties in the research location

\begin{tabular}{|c|c|c|c|c|c|c|c|c|c|c|c|c|}
\hline Treatment & $\begin{array}{l}\mathrm{pH} \\
\mathrm{H}_{2} \mathrm{O}\end{array}$ & $\begin{array}{l}\mathrm{pH} \\
\mathrm{KCl}\end{array}$ & $\begin{array}{l}\mathrm{C} \\
\text { Organic }\end{array}$ & $\begin{array}{l}\mathrm{C} / \mathrm{N} \\
\text { Ratio (\%) }\end{array}$ & $\begin{array}{l}\mathrm{N} \\
\text { Total }\end{array}$ & $\begin{array}{l}P \\
\text { available } \\
(\text { ppm) }\end{array}$ & & $\mathrm{Ca}$ & $\begin{array}{l}\mathrm{Mg} \\
\text { me } 100 \\
\mathrm{~g}^{-1} \text { soil }\end{array}$ & CEC & $\begin{array}{l}\text { Exch } \\
\mathrm{Al}\end{array}$ & Exch-H \\
\hline K0R1 & $4.92 \mathrm{abc}$ & $4.65 \mathrm{ab}$ & $1.07 \mathrm{a}$ & $10.43 \mathrm{abc}$ & $0.10 \mathrm{a}$ & $48.64 \mathrm{a}$ & $0.09 \mathrm{bc}$ & $1.64 \mathrm{ab}$ & $0.31 \mathrm{abc}$ & $3.87 \mathrm{abc}$ & $0.49 \mathrm{a}$ & $0.28 \mathrm{~b}$ \\
\hline K0R2 & $3.94 \mathrm{c}$ & $3.85 \mathrm{~b}$ & $0.58 \mathrm{~b}$ & $12.14 \mathrm{a}$ & $0.05 \mathrm{~b}$ & $5.56 \mathrm{c}$ & $0.03 \mathrm{c}$ & $0.40 \mathrm{~b}$ & $0.15 \mathrm{c}$ & $2.14 \mathrm{~d}$ & $0.51 \mathrm{a}$ & $0.17 \mathrm{~b}$ \\
\hline K1R1 & $5.09 \mathrm{abc}$ & $4.80 \mathrm{ab}$ & $1.18 \mathrm{a}$ & $11.62 \mathrm{a}$ & $0.10 \mathrm{a}$ & $52.62 \mathrm{a}$ & $0.17 \mathrm{a}$ & $1.85 \mathrm{ab}$ & $0.35 \mathrm{abc}$ & $4.26 \mathrm{ab}$ & $0.15 \mathrm{ab}$ & $0.27 \mathrm{~b}$ \\
\hline K1R2 & $4.29 \mathrm{bc}$ & $4.24 \mathrm{ab}$ & $0.52 \mathrm{~b}$ & $8.21 \mathrm{c}$ & $0.06 \mathrm{~b}$ & $10.78 \mathrm{c}$ & $0.04 \mathrm{c}$ & $0.92 \mathrm{ab}$ & $0.28 \mathrm{bc}$ & $2.13 \mathrm{~d}$ & $0.18 \mathrm{ab}$ & $0.36 \mathrm{~b}$ \\
\hline K2R1 & $5.15 \mathrm{abc}$ & $4.89 \mathrm{ab}$ & $1.14 \mathrm{a}$ & $12.41 \mathrm{a}$ & $0.09 \mathrm{a}$ & $37.81 \mathrm{ab}$ & $0.09 \mathrm{bc}$ & $1.91 \mathrm{ab}$ & $0.40 \mathrm{abc}$ & $4.42 \mathrm{ab}$ & $0.03 \mathrm{~b}$ & $0.29 \mathrm{~b}$ \\
\hline $\mathrm{K} 2 \mathrm{R} 2$ & $4.65 \mathrm{abc}$ & $4.39 \mathrm{ab}$ & $0.49 \mathrm{~b}$ & $8.13 \mathrm{c}$ & $0.06 \mathrm{~b}$ & $9.77 \mathrm{c}$ & $0.06 \mathrm{bc}$ & $1.07 \mathrm{ab}$ & $0.30 \mathrm{bc}$ & $2.35 \mathrm{~cd}$ & $0.26 \mathrm{ab}$ & $0.40 \mathrm{ab}$ \\
\hline K3R1 & $5.21 \mathrm{abc}$ & $5.02 \mathrm{ab}$ & $1.04 \mathrm{a}$ & $10.41 \mathrm{abc}$ & $0.10 \mathrm{a}$ & $56.50 \mathrm{a}$ & $0.13 \mathrm{ab}$ & $1.99 \mathrm{ab}$ & $0.45 \mathrm{abc}$ & $4.93 \mathrm{a}$ & $\mathrm{ND} b$ & $0.32 \mathrm{~b}$ \\
\hline K3R2 & $5.04 \mathrm{abc}$ & $4.86 \mathrm{ab}$ & $0.51 \mathrm{~b}$ & $11.04 \mathrm{ab}$ & $0.05 \mathrm{~b}$ & $13.95 \mathrm{bc}$ & $0.04 \mathrm{c}$ & $1.08 \mathrm{ab}$ & $0.38 \mathrm{abc}$ & $3.33 \mathrm{abcd}$ & $0.08 \mathrm{ab}$ & $0.43 \mathrm{ab}$ \\
\hline K4R1 & $5.23 \mathrm{ab}$ & $5.09 \mathrm{ab}$ & $1.10 \mathrm{a}$ & $10.95 \mathrm{ab}$ & $0.10 \mathrm{a}$ & $50.32 \mathrm{a}$ & $0.09 \mathrm{bc}$ & $2.14 \mathrm{a}$ & $0.55 \mathrm{ab}$ & $4.40 \mathrm{ab}$ & $\mathrm{ND} b$ & $0.35 \mathrm{~b}$ \\
\hline K4R2 & $5.10 \mathrm{abc}$ & $4.88 \mathrm{ab}$ & $0.51 \mathrm{~b}$ & $8.77 \mathrm{bc}$ & $0.06 \mathrm{~b}$ & $14.23 \mathrm{bc}$ & $0.05 \mathrm{c}$ & $1.32 \mathrm{ab}$ & $0.40 \mathrm{abc}$ & $2.80 \mathrm{bcd}$ & $\mathrm{ND} b$ & $0.55 \mathrm{ab}$ \\
\hline K5R1 & $5.42 \mathrm{ab}$ & $5.15 \mathrm{ab}$ & $1.08 \mathrm{a}$ & $12.25 \mathrm{a}$ & $0.09 \mathrm{a}$ & $58.96 \mathrm{a}$ & $0.19 \mathrm{a}$ & $2.21 \mathrm{a}$ & $0.62 \mathrm{a}$ & $4.42 \mathrm{ab}$ & $\mathrm{ND} b$ & $0.53 \mathrm{ab}$ \\
\hline $\mathrm{K} 5 \mathrm{R} 2$ & $5.65 \mathrm{a}$ & $5.43 \mathrm{a}$ & $0.59 \mathrm{~b}$ & $10.41 \mathrm{abc}$ & $0.06 \mathrm{~b}$ & $12.49 \mathrm{bc}$ & $0.04 \mathrm{c}$ & $1.81 \mathrm{ab}$ & $0.54 \mathrm{ab}$ & $2.86 \mathrm{bcd}$ & $\mathrm{ND} b$ & $0.78 \mathrm{a}$ \\
\hline R1 & $5.17 \mathrm{a}$ & $4.94 \mathrm{a}$ & $1.10 \mathrm{a}$ & $11.34 \mathrm{a}$ & $0.10 \mathrm{a}$ & $50.81 \mathrm{a}$ & $0.13 \mathrm{a}$ & $1.95 \mathrm{a}$ & $0.45 \mathrm{a}$ & $4.38 \mathrm{a}$ & $0.11 \mathrm{a}$ & $0.34 \mathrm{a}$ \\
\hline R2 & $4.78 \mathrm{a}$ & $4.61 \mathrm{a}$ & $0.54 \mathrm{~b}$ & $9.78 \mathrm{~b}$ & $0.06 \mathrm{~b}$ & $11.13 \mathrm{~b}$ & $0.04 \mathrm{~b}$ & $1.10 \mathrm{~b}$ & $0.34 \mathrm{a}$ & $2.60 \mathrm{~b}$ & $0.17 \mathrm{a}$ & $0.45 \mathrm{a}$ \\
\hline K0 & $4.43 \mathrm{~b}$ & $4.25 \mathrm{~b}$ & $0.83 \mathrm{a}$ & $11.28 \mathrm{a}$ & $0.08 \mathrm{a}$ & $27.10 \mathrm{a}$ & $0.06 \mathrm{a}$ & $1.02 \mathrm{a}$ & $0.23 \mathrm{c}$ & $3.01 \mathrm{a}$ & $0.50 \mathrm{a}$ & $0.23 \mathrm{~b}$ \\
\hline K1 & $4.69 \mathrm{ab}$ & $4.52 \mathrm{ab}$ & $0.85 \mathrm{a}$ & $9.91 \mathrm{a}$ & $0.08 \mathrm{a}$ & $31.70 \mathrm{a}$ & $0.10 \mathrm{a}$ & $1.39 \mathrm{a}$ & $0.31 b c$ & $3.20 \mathrm{a}$ & $0.16 \mathrm{~b}$ & $0.32 \mathrm{~b}$ \\
\hline K2 & $4.90 \mathrm{ab}$ & $4.64 \mathrm{ab}$ & $0.82 \mathrm{a}$ & $10.27 \mathrm{a}$ & $0.08 \mathrm{a}$ & $23.79 \mathrm{a}$ & $0.08 \mathrm{a}$ & $1.49 \mathrm{a}$ & $0.35 \mathrm{bc}$ & $3.38 \mathrm{a}$ & $0.14 b$ & $0.34 \mathrm{~b}$ \\
\hline K3 & $5.12 \mathrm{ab}$ & $4.94 \mathrm{ab}$ & $0.78 \mathrm{a}$ & $10.73 \mathrm{a}$ & $0.07 \mathrm{a}$ & $35.22 \mathrm{a}$ & $0.08 \mathrm{a}$ & $1.53 \mathrm{a}$ & $0.41 \mathrm{abc}$ & $4.13 \mathrm{a}$ & $0.04 \mathrm{~b}$ & $0.37 \mathrm{~b}$ \\
\hline K4 & $5.17 \mathrm{ab}$ & $4.97 \mathrm{ab}$ & $0.81 \mathrm{a}$ & $9.86 \mathrm{a}$ & $0.08 \mathrm{a}$ & $32.28 \mathrm{a}$ & $0.07 \mathrm{a}$ & $1.73 \mathrm{a}$ & $0.48 \mathrm{ab}$ & $3.60 \mathrm{a}$ & $\mathrm{ND} b$ & $0.45 \mathrm{ab}$ \\
\hline K5 & $5.53 \mathrm{a}$ & $5.29 \mathrm{a}$ & $0.83 \mathrm{a}$ & $11.33 \mathrm{a}$ & $0.07 \mathrm{a}$ & $35.73 \mathrm{a}$ & $0.11 \mathrm{a}$ & $2.01 \mathrm{a}$ & $0.58 \mathrm{a}$ & $3.64 \mathrm{a}$ & $\mathrm{ND} b$ & $0.65 \mathrm{a}$ \\
\hline Interaction & + & + & + & + & + & + & + & + & + & + & + & + \\
\hline
\end{tabular}

Note: Numbers followed by the same letter in the same column does not differ significantly at duncant's multiple range test (DMRT) and $(+)$ mark indicates there is an interaction to the combination treatment.

dolomite of immersion depth with $6,000 \mathrm{~kg} \mathrm{ha}^{-1}$ dose can increase $\mathrm{pH}$ from 4.92 to 5.42 ( 0.5 increased). The $\mathrm{pH} \mathrm{H}_{2} \mathrm{O}$ in $20-40 \mathrm{~cm}$ of immersion depth increased by 1.71 from 3.94 (very acidic) to 5.65 (moderate acidic).

Soil supporting capability to $\mathrm{pH}$ in $0-20 \mathrm{~cm}$ of depth was higher than $20-40 \mathrm{~cm}$ of depth. It was because of higher scores of cation-exchange capability (CEC) and organic-C in those layers.

Foller et al. (1981) suggested that smaller dolomite dose provide faster reaction. A very fine size which was $100 \%$ passing 100 mesh would provide faster effect compared to $90 \%$ passing 10 mesh. However, fine sizes will be easier to be washed making them not last longer compared to coarser sizes. Finer sizes of dolomite will be more difficult to be immersed by implementer and the price will also increase.

Organic-C content analysis results showed that the dolomite doses did not show significant difference, but immersion depths showed varying significant different scores of organic-C. This was possibly due to the chopping process of sugarcane litter, stump, and plant (brushing in the land processing) which took an important role in the varying organic-C content in each treatment. Plowing in the land processing also gave an important role in providing organic material content.

Dolomite additional doses did not have significant effect, but dolomite immersion depths had significant effect to total $\mathrm{N}$, available $\mathrm{P}$ and $\mathrm{K}$, with higher score at each parameter which was obtained at $0-20 \mathrm{~cm}$ of immersion depth. The reason was that the analysis result showed higher scores of $\mathrm{pH}$ in $0-20 \mathrm{~cm}$ of soil layer depth, so that the soil nutrient availabilities improved and this was supported by higher score of cation-exchange capability in these layers.

Exchangeable calcium in each layer increased along with dolomite dose addition. Calcium in $0-20$ $\mathrm{cm}$ of depth increased by $0.57 \mathrm{me}_{100 \mathrm{~g}^{-1}}$ soil (K0R1

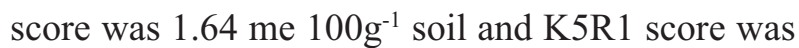

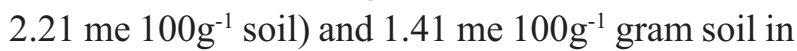
$20-40 \mathrm{~cm}$ of depth (K0 R2 score was $0.40 \mathrm{me}^{100 \mathrm{~g}^{-1}}$ soil with very low category and K5 score was 1.81 me $100 \mathrm{~g}^{-1}$ soil with low category). The calcium 
Table 4. Leaf nutrient content

\begin{tabular}{|c|c|c|c|c|c|}
\hline Treatment & N (\%) & $\mathrm{P}(\%)$ & $\mathrm{K}(\%)$ & $\mathrm{Ca}(\%)$ & $\operatorname{Mg}(\%)$ \\
\hline$\overline{\text { K0R1 }}$ & $1.86 \mathrm{~b}$ & $0.31 \mathrm{a}$ & $1.16 \mathrm{a}$ & $0.189 \mathrm{ab}$ & $0.12 b c$ \\
\hline K0R2 & $1.89 \mathrm{ab}$ & $0.31 \mathrm{a}$ & $1.18 \mathrm{a}$ & $0.178 \mathrm{~b}$ & $0.11 \mathrm{c}$ \\
\hline K1R1 & $1.88 \mathrm{ab}$ & $0.32 \mathrm{a}$ & $1.19 \mathrm{a}$ & $0.187 \mathrm{ab}$ & $0.13 \mathrm{abc}$ \\
\hline K1R2 & $2.13 \mathrm{ab}$ & $0.32 \mathrm{a}$ & $1.21 \mathrm{a}$ & $0.180 \mathrm{ab}$ & $0.13 \mathrm{abc}$ \\
\hline K2R1 & $2.05 \mathrm{ab}$ & $0.31 \mathrm{a}$ & $1.15 \mathrm{a}$ & $0.190 \mathrm{ab}$ & $0.13 \mathrm{abc}$ \\
\hline K2R2 & $2.03 \mathrm{ab}$ & $0.31 \mathrm{a}$ & $1.20 \mathrm{a}$ & $0.189 \mathrm{ab}$ & $0.12 b c$ \\
\hline K3R1 & $2.03 \mathrm{ab}$ & $0.31 \mathrm{a}$ & $1.17 \mathrm{a}$ & $0.192 \mathrm{ab}$ & $0.13 \mathrm{abc}$ \\
\hline K3R2 & $2.12 \mathrm{ab}$ & $0.30 \mathrm{a}$ & $1.19 \mathrm{a}$ & $0.199 \mathrm{ab}$ & $0.14 \mathrm{ab}$ \\
\hline K4R1 & $1.96 \mathrm{ab}$ & $0.31 \mathrm{a}$ & $1.16 \mathrm{a}$ & $0.203 \mathrm{ab}$ & $0.15 \mathrm{a}$ \\
\hline K4R2 & $2.10 \mathrm{ab}$ & $0.33 \mathrm{a}$ & $1.18 \mathrm{a}$ & $0.197 \mathrm{ab}$ & $0.13 a b c$ \\
\hline K5R1 & $2.05 \mathrm{ab}$ & $0.31 \mathrm{a}$ & $1.23 \mathrm{a}$ & $0.205 \mathrm{a}$ & $0.13 \mathrm{abc}$ \\
\hline K5R2 & $2.14 \mathrm{a}$ & $0.32 \mathrm{a}$ & $1.19 \mathrm{a}$ & $0.199 \mathrm{ab}$ & $0.15 \mathrm{a}$ \\
\hline $\mathrm{R} 1$ & $1.97 \mathrm{a}$ & $0.31 \mathrm{a}$ & $1.18 \mathrm{a}$ & $0.194 \mathrm{a}$ & $0.13 \mathrm{a}$ \\
\hline $\mathrm{R} 2$ & $2.07 \mathrm{a}$ & $0.31 \mathrm{a}$ & $1.19 \mathrm{a}$ & $0.190 \mathrm{a}$ & $0.13 \mathrm{a}$ \\
\hline K0 & $1.88 \mathrm{~b}$ & $0.31 \mathrm{a}$ & $1.17 \mathrm{a}$ & $0.183 \mathrm{~b}$ & $0.12 \mathrm{~b}$ \\
\hline K1 & $2.01 \mathrm{ab}$ & $0.32 \mathrm{a}$ & $1.20 \mathrm{a}$ & $0.184 \mathrm{~b}$ & $0.13 \mathrm{ab}$ \\
\hline $\mathrm{K} 2$ & $2.04 \mathrm{ab}$ & $0.31 \mathrm{a}$ & $1.18 \mathrm{a}$ & $0.189 \mathrm{ab}$ & $0.13 \mathrm{ab}$ \\
\hline K3 & $2.08 \mathrm{a}$ & $0.31 \mathrm{a}$ & $1.18 \mathrm{a}$ & $0.196 \mathrm{ab}$ & $0.14 \mathrm{a}$ \\
\hline K4 & $2.03 \mathrm{ab}$ & $0.32 \mathrm{a}$ & $1.17 \mathrm{a}$ & $0.200 \mathrm{ab}$ & $0.14 \mathrm{a}$ \\
\hline K5 & $2.10 \mathrm{a}$ & $0.32 \mathrm{a}$ & $1.21 \mathrm{a}$ & $0.202 \mathrm{a}$ & $0.14 \mathrm{a}$ \\
\hline Interaction & + & - & - & + & + \\
\hline
\end{tabular}

increased in the lower soil layer was higher because sugarcane uptakes in the upper layer was higher and there was fine sized dolomite leaching due to the irrigation and rain.

The analysis results showed that increase of dolomite dose significantly affected the $\mathrm{Mg}$ availability, but the dolomite immersion depths did not. Exchangeable $\mathrm{Mg}$ at $0-20 \mathrm{~cm}$ increased by 0.31

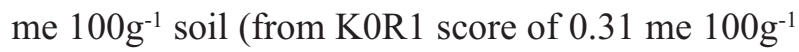
soil to K5R1 score of $0.62 \mathrm{me} 100 \mathrm{~g}^{-1}$ soil) and $\mathrm{H}^{+}$, it

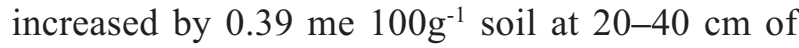
depth (from K0R2 score of 0.5 me $100 \mathrm{~g}^{-1}$ soil with very low category to K5R2 score of 0.54 me $100 \mathrm{~g}^{-1}$ soil with low category).

Table 3 shows the analysis of variance of exchangeable $\mathrm{Al}$ and exchangeable $\mathrm{H}$ in varying dolomite doses which showed significant difference, but dolomite immersion depths did not show significant difference. Table 3 suggests that the higher dolomite dose will decrease Al-exc score into very low and increase Hexc score. This was in accordance with Kamprath's (1967) suggesting that liming of acidic soil decrease aluminum concentration in the soil solution through deposition od $\mathrm{Al}_{3}{ }^{+}$into $\mathrm{Al}(\mathrm{OH})_{3}$, by the following reaction.

$$
\begin{aligned}
& \text { Misel } \mathrm{H}^{+}+2 \mathrm{CaCO}_{3}+3 \mathrm{H}_{2} \mathrm{O} \rightarrow \text { Misel } \mathrm{Ca}^{2+}+\mathrm{H}_{2} \mathrm{CO}_{3}+\mathrm{Al}(\mathrm{OH})_{3} \mathrm{\triangle} \\
& \mathrm{Al}^{3+} \\
& \mathrm{Ca}^{2+} \mathrm{KY} \\
& \mathrm{H}_{2} \mathrm{O} \quad \mathrm{H}_{2} \mathrm{O}
\end{aligned}
$$

The Al-exc score in 20-40 cm sof oil depth was higher because Al-exc would appear when the soil pH was acidic and soil pH scored in $20-40 \mathrm{~cm}$ of depth was lower than upper soil layer. 4,000 kg ha dolomite dose (K4) was able to decrease Al-exc $0.50 \mathrm{me} 100 \mathrm{~g}^{-1}$ soil (until it was undetected by the instrument). The increasing of $\mathrm{H}$-exc by $0.42 \mathrm{me}$ $100 \mathrm{~g}^{-1}$ soil (from K0 score of $0.23 \mathrm{me}^{100 \mathrm{~g}^{-1}}$ soil to $\mathrm{K} 5$ score of $0.65 \mathrm{me} 100 \mathrm{~g}^{-1}$ soil) along with the increasing of dolomite dose will cause higher neutralization, so that $\mathrm{H}$-exc will be more improved.

\section{Leaf nutrient content}

Table 4 shows that dolomite addition significantly affected the leaf $\mathrm{N}$ content. The highest leaf $\mathrm{N}$ of $2.08 \%$ and $2.10 \%$ was obtained at $2,000 \mathrm{~kg} \mathrm{ha}^{-1}$ and 
Table 5. Observation results on the sugarcane performance at 7 MAA and Ton Cane Hectare (TCH) at 12 MAA

\begin{tabular}{|c|c|c|c|c|c|c|c|c|c|c|c|}
\hline \multirow[b]{2}{*}{ Treatments } & \multirow{2}{*}{$\begin{array}{l}\text { Height } \\
(\mathrm{cm})\end{array}$} & \multirow{2}{*}{$\begin{array}{l}\text { Population } \\
\text { (ha) }\end{array}$} & \multicolumn{4}{|c|}{ Wet biomass (ton ha-1) } & \multicolumn{4}{|c|}{ Dry biomass (ton ha' ${ }^{-1}$ ) } & \multirow{2}{*}{$\begin{array}{l}\text { TCH } \\
(\text { ton ha-1) }\end{array}$} \\
\hline & & & Stem & $\begin{array}{l}\text { Green } \\
\text { leaf }\end{array}$ & $\begin{array}{l}\text { Dry } \\
\text { leaf }\end{array}$ & Root & Stem & $\begin{array}{l}\text { Green } \\
\text { leaf }\end{array}$ & $\begin{array}{l}\text { Dry } \\
\text { leaf }\end{array}$ & Root & \\
\hline K0R1 & $209.17 \mathrm{~b}$ & & $63.24 \mathrm{a}$ & $17.03 \mathrm{a}$ & $4.60 \mathrm{ab}$ & $2.97 \mathrm{ab}$ & $12.07 \mathrm{a}$ & $4.69 \mathrm{a}$ & $3.08 \mathrm{a}$ & $0.96 \mathrm{a}$ & $106.99 \mathrm{a}$ \\
\hline K0R2 & $203.00 \mathrm{~b}$ & $114.95 \mathrm{abc}$ & $57.11 \mathrm{a}$ & $17.21 \mathrm{a}$ & $4.23 \mathrm{ab}$ & $3.06 \mathrm{ab}$ & $11.18 \mathrm{a}$ & $4.61 \mathrm{a}$ & $2.54 \mathrm{a}$ & $0.99 \mathrm{a}$ & $103.63 \mathrm{a}$ \\
\hline K1R1 & $211.17 \mathrm{ab}$ & $104.50 \mathrm{bcd}$ & 54.59 a & $16.58 \mathrm{a}$ & $4.05 \mathrm{ab}$ & $2.97 \mathrm{ab}$ & $11.33 \mathrm{a}$ & $4.73 \mathrm{a}$ & $2.80 \mathrm{a}$ & $1.00 \mathrm{a}$ & $107.17 \mathrm{a}$ \\
\hline K1R2 & $210.00 \mathrm{ab}$ & $118.55 \mathrm{ab}$ & $55.58 \mathrm{a}$ & $17.03 \mathrm{a}$ & $4.50 \mathrm{ab}$ & $2.57 \mathrm{~b}$ & $11.06 \mathrm{a}$ & $4.71 \mathrm{a}$ & $3.06 \mathrm{a}$ & $0.87 \mathrm{a}$ & $102.30 \mathrm{a}$ \\
\hline K2R1 & $213.33 \mathrm{ab}$ & $126.45 \mathrm{a}$ & $60.81 \mathrm{a}$ & 20.18 a & $5.22 \mathrm{ab}$ & $3.33 \mathrm{ab}$ & $12.65 \mathrm{a}$ & $5.30 \mathrm{a}$ & $3.36 \mathrm{a}$ & $0.99 \mathrm{a}$ & $115.13 \mathrm{a}$ \\
\hline $\mathrm{K} 2 \mathrm{R} 2$ & $202.83 \mathrm{~b}$ & $117.47 \mathrm{ab}$ & $61.17 \mathrm{a}$ & $18.92 \mathrm{a}$ & $3.65 \mathrm{~b}$ & $3.51 \mathrm{ab}$ & $12.22 \mathrm{a}$ & $5.13 \mathrm{a}$ & $2.23 \mathrm{a}$ & $1.08 \mathrm{a}$ & $107.47 \mathrm{a}$ \\
\hline K3R1 & $213.67 \mathrm{ab}$ & $117.83 \mathrm{ab}$ & $67.11 \mathrm{a}$ & $17.03 \mathrm{a}$ & $4.23 \mathrm{ab}$ & $3.60 \mathrm{ab}$ & $12.82 \mathrm{a}$ & $4.58 \mathrm{a}$ & $2.56 \mathrm{a}$ & $0.99 \mathrm{a}$ & $128.49 \mathrm{a}$ \\
\hline K3R2 & $206.83 \mathrm{~b}$ & $96.21 \mathrm{~d}$ & $59.09 \mathrm{a}$ & $21.17 \mathrm{a}$ & $4.68 \mathrm{ab}$ & $3.69 \mathrm{ab}$ & $12.14 \mathrm{a}$ & $5.73 \mathrm{a}$ & $2.87 \mathrm{a}$ & $1.01 \mathrm{a}$ & $128.82 \mathrm{a}$ \\
\hline K4R1 & $223.00 \mathrm{a}$ & $111.70 \mathrm{abcd}$ & $58.28 \mathrm{a}$ & $20.54 \mathrm{a}$ & $4.86 \mathrm{ab}$ & $3.78 \mathrm{ab}$ & $11.60 \mathrm{a}$ & $5.31 \mathrm{a}$ & $2.82 \mathrm{a}$ & $1.10 \mathrm{a}$ & $132.50 \mathrm{a}$ \\
\hline K4R2 & $202.50 \mathrm{~b}$ & $114.77 \mathrm{abc}$ & $67.92 \mathrm{a}$ & $19.91 \mathrm{a}$ & $4.95 \mathrm{ab}$ & $3.78 \mathrm{ab}$ & $13.59 \mathrm{a}$ & $5.37 \mathrm{a}$ & $2.92 \mathrm{a}$ & $1.17 \mathrm{a}$ & $131.93 \mathrm{a}$ \\
\hline K5R1 & $209.83 \mathrm{ab}$ & $118.91 \mathrm{ab}$ & $71.89 \mathrm{a}$ & $22.70 \mathrm{a}$ & $4.14 \mathrm{ab}$ & $4.23 \mathrm{a}$ & $13.09 \mathrm{a}$ & $5.76 \mathrm{a}$ & $2.40 \mathrm{a}$ & $1.24 \mathrm{a}$ & $164.84 \mathrm{a}$ \\
\hline $\mathrm{K} 5 \mathrm{R} 2$ & $212.83 \mathrm{ab}$ & $112.42 \mathrm{abcd}$ & $62.88 \mathrm{a}$ & $20.18 \mathrm{a}$ & $5.72 \mathrm{a}$ & $3.42 \mathrm{ab}$ & $12.44 \mathrm{a}$ & $5.35 \mathrm{a}$ & $3.31 \mathrm{a}$ & $1.09 \mathrm{a}$ & $143.31 \mathrm{a}$ \\
\hline R1 & $213.36 \mathrm{a}$ & $112.54 \mathrm{a}$ & $62.65 \mathrm{a}$ & $19.00 \mathrm{a}$ & $4.52 \mathrm{a}$ & $3.48 \mathrm{a}$ & $12.26 \mathrm{a}$ & $5.06 \mathrm{a}$ & $2.84 \mathrm{a}$ & $1.05 \mathrm{a}$ & $125.85 \mathrm{a}$ \\
\hline $\mathrm{R} 2$ & $206.33 \mathrm{~b}$ & $112.39 \mathrm{a}$ & $60.63 \mathrm{a}$ & $19.07 \mathrm{a}$ & $4.62 \mathrm{a}$ & $3.34 \mathrm{a}$ & $12.10 \mathrm{a}$ & $5.15 \mathrm{a}$ & $2.82 \mathrm{a}$ & $1.04 \mathrm{a}$ & $119.58 \mathrm{a}$ \\
\hline K0 & $206.08 \mathrm{a}$ & $105.40 \mathrm{c}$ & $60.18 \mathrm{a}$ & $17.12 \mathrm{ab}$ & $4.41 \mathrm{a}$ & & $11.63 \mathrm{a}$ & $4.65 \mathrm{a}$ & $2.81 \mathrm{a}$ & $0.98 \mathrm{a}$ & $105.31 \mathrm{a}$ \\
\hline K1 & $210.58 \mathrm{a}$ & $111.52 \mathrm{bc}$ & $55.09 \mathrm{a}$ & $16.80 \mathrm{~b}$ & $4.28 \mathrm{a}$ & $2.77 \mathrm{a}$ & $11.19 \mathrm{a}$ & $4.72 \mathrm{a}$ & $2.93 \mathrm{a}$ & $0.93 \mathrm{a}$ & $104.74 \mathrm{a}$ \\
\hline K2 & $208.08 \mathrm{a}$ & $121.97 \mathrm{a}$ & $60.97 \mathrm{a}$ & $19.55 \mathrm{ab}$ & $4.44 \mathrm{a}$ & $3.42 \mathrm{a}$ & $12.43 \mathrm{a}$ & $5.21 \mathrm{a}$ & $2.79 \mathrm{a}$ & $1.04 \mathrm{a}$ & $111.30 \mathrm{a}$ \\
\hline K3 & $210.25 \mathrm{a}$ & $107.02 \mathrm{bc}$ & $63.10 \mathrm{a}$ & $19.10 \mathrm{ab}$ & $4.46 \mathrm{a}$ & $3.65 \mathrm{a}$ & $12.48 \mathrm{a}$ & $5.16 \mathrm{a}$ & $2.72 \mathrm{a}$ & $1.00 \mathrm{a}$ & $128.66 \mathrm{a}$ \\
\hline K4 & $212.75 \mathrm{a}$ & $113.23 \mathrm{bc}$ & $63.10 \mathrm{a}$ & $20.22 \mathrm{ab}$ & $4.91 \mathrm{a}$ & $3.78 \mathrm{a}$ & $12.60 \mathrm{a}$ & $5.34 \mathrm{a}$ & $2.87 \mathrm{a}$ & $1.13 \mathrm{a}$ & $132.21 \mathrm{a}$ \\
\hline K5 & $211.33 \mathrm{a}$ & $115.67 \mathrm{ab}$ & $67.38 \mathrm{a}$ & $21.44 \mathrm{a}$ & $4.93 \mathrm{a}$ & $3.83 \mathrm{a}$ & $12.76 \mathrm{a}$ & $5.56 \mathrm{a}$ & $2.86 \mathrm{a}$ & $1.17 \mathrm{a}$ & $154.07 \mathrm{a}$ \\
\hline
\end{tabular}

Interaction +

Note: Numbers followed by the same letter in the same column does not differ significantly at duncant's multiple range test (DMRT) and $(+)$ mark indicates there is an interaction to the combination treatment.

$6,000 \mathrm{~kg} \mathrm{ha}^{-1}$ of dolomite doses. Dolomite dose more than $2,000 \mathrm{~kg} \mathrm{ha}^{-1}$ would not provide significant effect to leaf nitrogen content.

According to Calcino (1995), the threshold of nitrogen nutrient in sugarcane leaf experienced deficit of $0.18 \%$. The lowest score of nitrogen nutrient content was obtained at the control (without dolomite treatment) with score of $0.188 \%$ and this score was above the deficiency score. Along with increasing dolomite dose the nitrogen content increased. For the crop year, it required dolomite addition or other sources of nitrogen so that sugarcane will not undergo nitrogen deficiency.

Table 4 shows that leaf phosphor (P) Content did not differ significantly from the doses and depths of dolomite placement. P content of leaf both in control and treatment contained of sufficient content of more than $0.19 \%$.

Potassium (K) content in the leaf in 7 months after application (MAA) of dolomite showed significant difference between treatments of dolomite doses and depths. Minimum potassium threshold content in sugarcane before the sugarcane was stated to be deficient of potassium was $1.11 \%$. All treatments showed that the potassium contents were above the threshold of potassium deficiency.

Table 4 shows that varying dolomite doses significantly affected the leaf potassium contents, but dolomite application depths did not significantly affected the leaf potassium contents.

There were interactions between doses and the depths of applications. The best interaction was obtained in the K5R1 treatment with $0.205 \%$ leaf $\mathrm{Ca}$ content score. The leaf nutrient contents analysis results showed that treatment $\mathrm{K} 0, \mathrm{~K} 1, \mathrm{~K} 2$ and $\mathrm{K} 3$ showed $\mathrm{Ca}$ deficiency. Minimum leaf $\mathrm{Ca}$ content was $0.2 \%$ and these four treatments had Ca content scores under threshold. $4,000 \mathrm{~kg} \mathrm{ha}^{-1}$ of dolomite addition was required to maintain sugarcane from Ca deficiency.

Table 5 shows that varying dolomite doses significantly affected the leaf $\mathrm{Mg}$ contents, but not the dolomite application depths. The lower Mg content was obtained at sugarcane without dolomite treatment. 
Table 6. Uptake and efficiency of calsium and magnesium in leaf

\begin{tabular}{|c|c|c|c|c|}
\hline \multirow{2}{*}{ Treatment } & \multicolumn{2}{|c|}{$\mathrm{Ca}$} & \multicolumn{2}{|c|}{$\mathrm{Mg}$} \\
\hline & Uptake & Efficiency & Uptake & Efficiency \\
\hline K0R1 & $14.76 \mathrm{a}$ & $0 \mathrm{a}$ & $9.28 \mathrm{abc}$ & $0 \mathrm{~b}$ \\
\hline K0R2 & $12.72 \mathrm{a}$ & $0 \mathrm{a}$ & $7.98 \mathrm{c}$ & $0 \mathrm{~b}$ \\
\hline K1R1 & $13.91 \mathrm{a}$ & $-0.79 a$ & $9.43 \mathrm{abc}$ & $0.31 \mathrm{~b}$ \\
\hline K1R2 & $13.99 \mathrm{a}$ & $1.18 \mathrm{a}$ & $9.85 \mathrm{abc}$ & $3.75 \mathrm{a}$ \\
\hline K2R1 & $16.42 \mathrm{a}$ & $0.77 \mathrm{a}$ & $11.61 \mathrm{abc}$ & $2.33 \mathrm{ab}$ \\
\hline K2R2 & $14.01 \mathrm{a}$ & $0.60 \mathrm{a}$ & $8.54 \mathrm{bc}$ & $0.56 \mathrm{ab}$ \\
\hline K3R1 & $13.63 \mathrm{a}$ & $-0.26 \mathrm{a}$ & $9.42 \mathrm{abc}$ & $0.07 \mathrm{~b}$ \\
\hline K3R2 & $17.16 \mathrm{a}$ & $1.03 \mathrm{a}$ & $12.40 \mathrm{ab}$ & $2.21 \mathrm{ab}$ \\
\hline K4R1 & $16.44 \mathrm{a}$ & $0.19 \mathrm{a}$ & $11.96 \mathrm{ab}$ & $0.67 \mathrm{ab}$ \\
\hline K4R2 & $16.15 \mathrm{a}$ & $0.39 \mathrm{a}$ & $10.74 \mathrm{abc}$ & $0.69 \mathrm{ab}$ \\
\hline K5R1 & $16.79 \mathrm{a}$ & $0.16 \mathrm{a}$ & $10.66 \mathrm{abc}$ & $0.23 \mathrm{ab}$ \\
\hline K5R2 & $17.27 \mathrm{a}$ & $0.35 \mathrm{a}$ & $12.82 \mathrm{a}$ & $0.81 \mathrm{ab}$ \\
\hline $\mathrm{R} 1$ & $15.32 \mathrm{a}$ & $0.01 \mathrm{a}$ & $10.39 \mathrm{a}$ & $0.60 \mathrm{a}$ \\
\hline $\mathrm{R} 2$ & $15.22 \mathrm{a}$ & $0.59 \mathrm{a}$ & $10.39 \mathrm{a}$ & $1.34 \mathrm{a}$ \\
\hline K0 & $13.74 \mathrm{a}$ & $0 \mathrm{a}$ & $8.63 \mathrm{~b}$ & $0 \mathrm{~b}$ \\
\hline K1 & $13.95 \mathrm{a}$ & $0.20 \mathrm{a}$ & $9.64 \mathrm{ab}$ & $2.03 \mathrm{a}$ \\
\hline K2 & $15.22 \mathrm{a}$ & $0.69 \mathrm{a}$ & $10.07 \mathrm{ab}$ & $1.45 \mathrm{a}$ \\
\hline K3 & $15.39 \mathrm{a}$ & $0.39 \mathrm{a}$ & $10.91 \mathrm{ab}$ & $1.14 \mathrm{a}$ \\
\hline K4 & $16.29 \mathrm{a}$ & $0.30 \mathrm{a}$ & $11.35 \mathrm{a}$ & $0.68 \mathrm{a}$ \\
\hline K5 & $17.03 \mathrm{a}$ & $0.26 \mathrm{a}$ & $11.74 \mathrm{a}$ & $0.52 \mathrm{a}$ \\
\hline
\end{tabular}

\begin{tabular}{llcc}
\hline Interaction & - & - & + \\
Note: Numbers followed by the same letter in the same column does not differ
\end{tabular} significantly at duncant's multiple range test (DMRT) and $(+)$ mark indicates there is an interaction to the combination treatment.

Dolomite doses from $2,000 \mathrm{~kg} \mathrm{ha}^{-1}$ to $6,000 \mathrm{~kg} \mathrm{ha}^{-1}$ showed highest $\mathrm{Mg}$ content and there was no significant difference among those three treatments. Dolomite dose above 2, $000 \mathrm{~kg} \mathrm{ha}^{-1}$ did not significantly affect sugarcane leaf $9 \mathrm{Mg}$ content. The deficiency threshold of leaf $\mathrm{Mg}$ content was $0.08 \%$. The analysis results of control and treatment groups did not show any $\mathrm{Mg}$ deficiency.

\section{Sugarcane growth}

The sugarcane population per hectare in 7 months after application (MAA) of varying dolomite doses had significant difference among treatments. The highest population was obtained at $1,000 \mathrm{~kg} \mathrm{ha}^{-1}$ of dolomite dose but it was not significantly different with $6,000 \mathrm{~kg} \mathrm{ha}^{-1}$ dose and it was significantly different without dolomite treatment. The possible reason was that the contents of nitrogen $(\mathrm{N})$, calcium $(\mathrm{Ca})$ and magnesium $(\mathrm{Mg})$ at dolomite dose treatments were higher than without dolomite treatment.

Sugarcane height in 7 months after application (MAA) was presented in Table 5. It is shown that the highest sugarcane height was obtained at $4,000 \mathrm{~kg}$ ha $^{-1}$ dolomite dose with $0-20 \mathrm{~cm}$ of application depth compared to without treatment in $20-40 \mathrm{~cm}$ of depth.

The measurement results to dry biomass of stem, fresh leaf, dry leaf and root of sugarcane at 7 MAA showed no significant effect among treatments of varying administered dolomite doses to wet biomass of stem, root, and green leaf. There were significant differences for dry sugarcane leaf biomass after application of 4,000 $\mathrm{kg} \mathrm{ha}^{-1}$ dolomite dose compared to control (without dolomite). In addition, there were interactions between dolomite doses and application of slot depths.

Table 5 shows that dry biomass of dry leaf with $1,000 \mathrm{~kg} \mathrm{ha}^{-1}$ and 4,000 $\mathrm{kg} \mathrm{ha}^{-1}$ of dolomite doses showed significant differences compared to control. There were no significant differences on dry biomass of stem, green leaf and root.

Table 5 shows that dolomite dose treatments at 0-20 $\mathrm{cm}$ and 20-40 $\mathrm{cm}$ of immersion depths showed no difference results from sugarcane TCH scores. 
The highest TCH was obtained in $6,000 \mathrm{~kg} \mathrm{ha}^{-1}$ of dolomite dose (K5) at 154.07 ton $\mathrm{ha}^{-1}$, while the lowest $\mathrm{TCH}$ was obtained at $500 \mathrm{~kg} \mathrm{ha}^{-1}$ of dolomite dose (K1) with 104.74 ton ha-1. $500 \mathrm{~kg} \mathrm{ha}^{-1}$ of dolomite dose showed no difference results compared to control. Increasing dolomite dose would increase TCH score. This was because more dolomite dose would improve soil chemical properties so as to improve soil nutrient uptakes as it was marked with significant differences of sugarcane leaf nutrient scores.

Dolomite dose and immersion and dolomite dose $6,000 \mathrm{~kg} \mathrm{ha}^{-1}$ showed depths of dolomite did not show score of $\mathrm{Ca}$ and $\mathrm{Mg}$ highest uptake. $\mathrm{Mg}$ interaction (Table 6). But treatment of 0-uptake showed interaction and treatment $20 \mathrm{~cm}$ of depth (R1) showed score of $\mathrm{Ca}$ combination showed significant uptake higher than $20-40 \mathrm{~cm}$ of depth (R2) differences

\section{CONCLUSIONS}

The dolomite immersion depths did not show significant effects to $\mathrm{N}, \mathrm{P}, \mathrm{K}, \mathrm{Ca}$ and $\mathrm{Mg}$ content in leaf. Dolomite addition show significant effects to $\mathrm{N}$ content in leaf. Dolomite addition significant effects to $\mathrm{K}$ content in leaf.

\section{REFERENCES}

Calcino, D.V. 1995. Australian sugar cane nutrition manual. Queensland: Bureau of Sugar Experiment Stations.

Follet, R.H., L.S. Murphy and R.L. Donahue. Fertilizers and soil amendments. New Jersey: Englewood Cliffs.

Gomez, K.A. dan A.A. Gomez. 1995. Prosedur statistik untuk penelitian pertanian. Diterjemahkan oleh: E. Sjamsuddin dan J.S. Baharsjah. Jakarta: UI-Press.

Kamprath, E. 1967. Soil acidity and response to liming, int. soil testing program tech. bull. 4. North Carolina State University: Raleigh.

Santoso E., Prihatini T., Prastowo, Komariah S. 1999. Peranan berbagai bahan sisa panen dan inokulan mikroba pada serapan hara, hasil padi, dan sifat kimia tanah. Prosiding Seminar Nasional Sumberdaya Tanah, Iklim dan Pupuk., Pusat Penelitian Tanah dan Agroklimat. Badan Penelitian dan Pengembangan Pertanian, Departemen Pertanian.

Sutanto, R. 2005. Dasar-dasar ilmu tanah. Yogyakarta: Kanisius.

Tambunan, W. A. 2008. Kajian sifat fisik dan kimia tanah hubungannya dengan produksi kelapa sawit (elaeis guineensis, jacq) di kebun kwala sawit ptpn ii. Tesis. Universitas Sumatera Utara. Medan. 